\title{
Dark exciton-exciton annihilation in monolayer $\mathrm{WSe}_{2}$
}

\author{
Daniel Erkensten $\odot,{ }^{1}$ Samuel Brem $\odot,{ }^{2}$ Koloman Wagner $\odot,{ }^{3,7}$ Roland Gillen $\odot,{ }^{4}$ Raül Perea-Causín $\odot,{ }^{1}$ Jonas D. Ziegler $\odot,{ }^{3,7}$

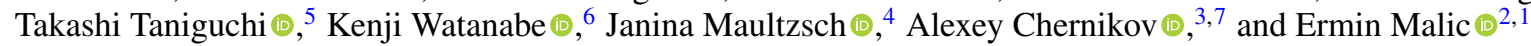 \\ ${ }^{1}$ Department of Physics, Chalmers University of Technology, 41296 Gothenburg, Sweden \\ ${ }^{2}$ Department of Physics, Philipps-Universität Marburg, 35037 Marburg, Germany \\ ${ }^{3}$ Department of Physics, University of Regensburg, D-93040 Regensburg, Germany \\ ${ }^{4}$ Department of Physics, Friedrich-Alexander-Universität Erlangen-Nürnberg, 91058 Erlangen-Nürnberg, Germany \\ ${ }^{5}$ International Center for Materials Nanoarchitectonics, National Institute for Materials Science, 1-1 Namiki, Tsukuba 305-004, Japan \\ ${ }^{6}$ Research Center for Functional Materials, National Institute for Materials Science, Tsukuba, 1-1 Namiki, Tsukuba 305-004, Japan \\ ${ }^{7}$ Dresden Integrated Center for Applied Physics and Photonic Materials (IAPP) and Würzburg-Dresden Cluster of Excellence ct.qmat, \\ Technische Universität Dresden, 01062 Dresden, Germany
}

(Received 16 June 2021; revised 25 November 2021; accepted 1 December 2021; published 17 December 2021)

The exceptionally strong Coulomb interaction in semiconducting transition-metal dichalcogenides (TMDs) gives rise to a rich exciton landscape consisting of bright and dark exciton states. At elevated densities, excitons can interact through exciton-exciton annihilation (EEA), an Auger-like recombination process limiting the efficiency of optoelectronic applications. Although EEA is a well-known and particularly important process in atomically thin semiconductors determining exciton lifetimes and affecting transport at elevated densities, its microscopic origin has remained elusive. In this joint theory-experiment study combining microscopic and material-specific theory with time- and temperature-resolved photoluminescence measurements, we demonstrate the key role of dark intervalley states that are found to dominate the EEA rate in monolayer $\mathrm{WSe}_{2}$. We reveal an intriguing, characteristic temperature dependence of Auger scattering in this class of materials with an excellent agreement between theory and experiment. Our study provides microscopic insights into the efficiency of technologically relevant Auger scattering channels within the remarkable exciton landscape of atomically thin semiconductors.

DOI: 10.1103/PhysRevB.104.L241406

\section{INTRODUCTION}

Atomically thin nanomaterials, such as transition-metal dichalcogenides (TMDs), offer an unprecedented platform to study intriguing many-particle phenomena in a broad range of external conditions [1-4]. The weak dielectric screening and the resulting strong Coulomb interaction in these materials give rise to the formation of tightly bound excitons and promote efficient interactions between charge carriers at elevated densities. In particular, excitons can interact through exciton-exciton annihilation (EEA), an Auger recombination process shown to be very efficient in TMDs [5-8]. EEA is a nonradiative scattering process, in which one exciton recombines nonradiatively by transferring its energy and momentum to another exciton, resulting in a highly excited electron-hole pair (HX) [9-11], cf. Fig. 1(a). The inverse process of impact excitation resulting in charge carrier multiplication has also been recently observed [12]. Auger recombination leads to an effective saturation of exciton densities [1] and is thus of crucial importance for the performance of many technological applications, such as photodetectors and solar cells.

Published by the American Physical Society under the terms of the Creative Commons Attribution 4.0 International license. Further distribution of this work must maintain attribution to the author(s) and the published article's title, journal citation, and DOI. Funded by Bibsam.
Auger scattering has previously been shown to be extremely efficient in graphene [13-16], but was initially considered to be inefficient in TMDs due to the difficulty to simultaneously conserve energy and momentum in parabolic band structures and the lack of resonant final states. However, recent up-converted photoluminescence (PL) measurements and $a b$ initio calculations confirmed the existence of a higher energetic exciton state appearing at approximately twice the $A$ exciton resonance both in monolayer and bilayer $\mathrm{WSe}_{2}$ $[10,17]$. This can be attributed to the existence of higherlying conduction bands, enabling a particular type of resonant Auger scattering $[9,18,19]$, cf. Fig. 1. In the regular intravalley Auger recombination process discussed so far in the literature, an optically excited carrier recombines with the hole at the $\mathrm{K}$ point and induces the excitation of another carrier into a higher conduction band [process I in Fig. 1(b)].

A microscopic understanding of Auger-like excitonexciton annihilation in atomically thin semiconductors in the entire exciton landscape is still lacking. Including just the regular intravalley Auger processes turns out to be far from sufficient to explain the large EEA rates measured across different TMD materials [5-8,20]. Phonon-assisted excitonelectron Auger recombination including dark states was also shown to be strongly suppressed [21] and cannot be responsible for the EEA efficiency seen in experiments. Furthermore, the recent observations of a strong substrate dependence of Auger scattering in TMD monolayers [22,23] still require a consistent explanation. Finally, even the bound or free nature 


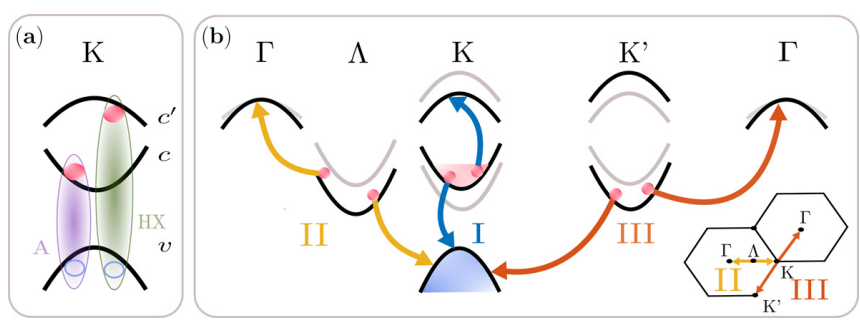

FIG. 1. Schematic illustration of exciton-exciton annihilation (EEA) channels in $\mathrm{WSe}_{2}$. (a) The annihilation of $A$ excitons (purple) gives rise to a higher-lying HX exciton state (green). (b) Regular intravalley (I blue) and additional intervalley Auger recombination processes (II orange and III red) involving momentum-dark $\mathrm{KK}^{\prime}$, $\mathrm{K} \Lambda$, and $\mathrm{K} \Gamma$ excitons, respectively. The spin-split conduction bands are distinguished by black and grey lines, respectively.

of the highly excited electron-hole pair that remains in the systems after EEA is not clear. Importantly, the rich excitonic landscape of TMDs consists not only of bright intravalley excitons but also of momentum-dark intervalley excitons with nonzero center-of-mass momenta [24,25]. These are expected to open up additional channels for Auger scattering that would satisfy momentum and energy conservation requirements. Moreover, since the energetically lowest states of tungsten-based TMDs are dark [24,26-28], one would expect intervalley exciton-exciton Auger recombination processes [II, III in Fig. 1(b)] to be particularly relevant.

In this joint theory-experiment study, we address the nature of Auger-like exciton-exciton annihilation in atomically thin semiconductors by combining time- and temperature-resolved PL measurements with material-specific microscopic modeling including density matrix and density functional theory methods. In particular, we investigate intra and intervalley Auger recombination channels in monolayer $\mathrm{WSe}_{2}$ for different substrates and temperatures. Crucially, we show that dark intervalley Auger recombination clearly dominates the exciton-exciton annihilation. We reveal an intriguing temperature dependence, characteristic for the impact of dark states-in excellent agreement between theory and experiment. Moreover, our calculations provide insight into the previously observed decrease of Auger scattering for hBNencapsulated $\mathrm{WSe}_{2}$ and $\mathrm{WS}_{2}$ monolayers [20,22,23] and we explain this effect with the changed resonance condition within the excitonic bandstructure.

\section{MODELING OF EXCITON-EXCITON ANNIHILATION RATES}

To develop a realistic and material-specific approach providing microscopic insights into exciton-exciton annihilation processes in TMD monolayers, we combine first-principle calculations with the excitonic density matrix formalism [28,29]. First, we define the many-particle Hamilton operator

$$
H_{x-x}=\frac{1}{2} \sum_{\substack{\mu \nu \rho \\ \mathbf{Q}, \mathbf{Q}^{\prime}}} W_{\mathbf{Q}, \mathbf{Q}^{\prime}}^{\mu \nu \rho} Y_{\mu, \mathbf{Q}+\mathbf{Q}^{\prime}}^{\dagger} X_{\nu, \mathbf{Q}} X_{\rho, \mathbf{Q}^{\prime}}+\text { H.c. }
$$

describing the annihilation of two excitons in the states $v$ and $\rho$ with center-of-mass momenta $\mathbf{Q}$ and $\mathbf{Q}^{\prime}$, respectively, which gives rise to the formation of a single higher energetic exciton in the state $\mu$. The later state can be generally considered to be either a ground or an excited state with a principal quantum number $n$ that could also include unbound electron-hole pairs in the limit of $n \rightarrow \infty$. We distinguish between excitons formed from the higher-lying conduction band $c^{\prime}$ and the valence band (in the following denoted as HX excitons) and regular spin-allowed $A$ excitons through the exciton creation operators $X^{\dagger}=c^{\dagger} v$ and $Y^{\dagger}=c^{\prime \dagger} v$, respectively, cf. Fig. 1(a). Here we emphasize that HX excitons can be generally formed by electrons and holes located at different high-symmetry points of the hexagonal Brillouin zone. The compound indices $\mu, v$, and $\rho$ include the excitonic spin, the principal quantum numbers $n=1 s, 2 s, \ldots$, and the excitonic valley $\xi=\left(\xi_{h} \xi_{e}\right)=\mathrm{KK}^{\left({ }^{\prime}\right)}, \mathrm{K} \boldsymbol{\Lambda}, \mathrm{K} \Gamma$, where the first (second) letter describes the valley in which the Coulomb-bound hole (electron) is localized. In this work, we consider the hole to be at the $\mathrm{K}$ point, but allow the electron to be at the $\mathrm{K}, \mathrm{K}^{\prime}, \Lambda$ or $\Gamma$ point, cf. Fig. 1(b).

The Auger matrix element $W_{\mathbf{Q}, Q^{\prime}}^{\mu \nu \rho}$ appearing in Eq. (1) determines the efficiency of the exciton-exciton annihilation process and consists of a direct and an exchange term, the exchange terms reflects the fermionic character of the excitons. The direct and exchange components of the matrix element, which is provided in Eq. (S11) in the Supplemental Material (SM) [30], crucially depend on the $A$ and HX exciton wave functions (obtained from the Wannier equation [31-33]) as well as the screened Coulomb interaction and electronic dipole matrix elements, with the matrix elements being extacted from $a b$ initio $\mathrm{G}_{0} \mathrm{~W}_{0}$ calculations. Additional details on the microscopic derivation of the Auger matrix elements and the $a b$ initio modeling are found in Secs. II A and III (SM), respectively.

Figure 2(a) illustrates the excitonic Auger matrix elements as a function of the principal quantum number $n$ of the final HX exciton state for the intra and intervalley processes I, II, and III in hBN-encapsulated $\mathrm{WSe}_{2}$ depicted in Fig. 1(b). Here, we evaluated the matrix elements for $\boldsymbol{Q}=\boldsymbol{Q}^{\prime}=0$, while the full momentum-dependent matrix elements are provided in SM, cf. Fig. S2. We find that, due to the large momentum transfer, the intervalley $\left(\xi_{v}=\xi_{\rho}=\mathrm{KK}^{\prime}, \xi_{\mu}=\mathrm{K} \Gamma\right)$ matrix element is up to two orders of magnitude smaller than the corresponding intravalley $\left(\xi_{v}=\xi_{\rho}=\xi_{\mu}=\mathrm{KK}\right)$ matrix element (note the logarithmic scale). Note that we restrict our calculations to $A$ excitons in the ground state $n=1 s$, and vary the principal quantum number of the HX exciton in Fig. 2(a). We find that the matrix elements decrease rapidly with increasing quantum index $n$ due to a shrinking momentum-space overlap with the initial $1 s$ state. The only exception is the intravalley $n=2 \mathrm{HX}$ state with a slightly larger coupling than for $n=1$. Most importantly, these results imply that the coupling to unbound electron-hole pairs in the limit of $n \rightarrow \infty$ should be small. Based on our microscopic model for the excitonic Auger matrix element, we now determine the exciton-exciton annihilation coefficient $R_{A}$.

The resulting experimentally accessible recombination rates lead to an effective saturation of exciton densities and are thus important for many technological devices based on TMDs. We exploit the Heisenberg's equation of motion to determine the temporal evolution of the density of 


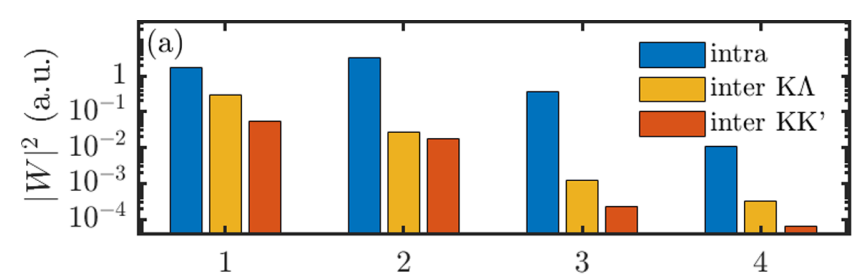

Final exciton state $n s$

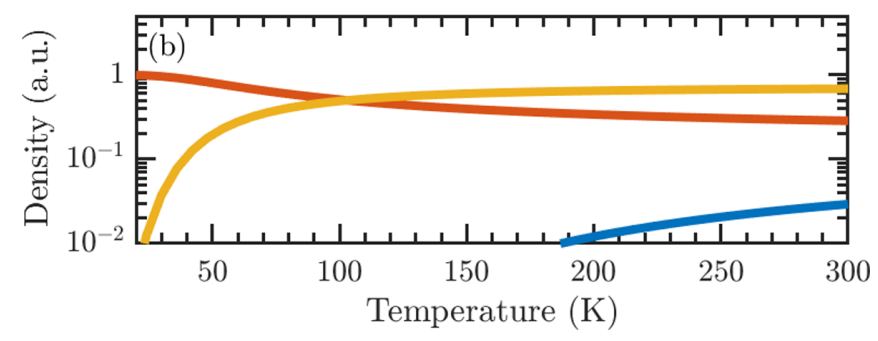

FIG. 2. Auger matrix elements $W$ and valley-specific exciton densities in $\mathrm{hBN}$-encapsulated $\mathrm{WSe}_{2}$. (a) Intravalley and intervalley exciton Auger matrix elements $|W|^{2}$ evaluated at $\mathbf{Q}=\mathbf{Q}^{\prime}=0$ and their dependence on the final (HX) state quantum index $n$. The matrix elements decrease rapidly with $n$ due to the small overlap between initial $1 s$ states and $n>1 s$ HX states. (b) Temperature-dependent valley-specific exciton densities in thermal equilibrium illustrating the crucial impact of intervalley $\mathrm{K} \Lambda$ (orange) and $\mathrm{KK}^{\prime}$ (red) excitons and weak impact of intravalley (blue) excitons.

$A$ excitons $n_{x}=\sum_{\nu \boldsymbol{Q}} N_{\mathrm{A}, \boldsymbol{Q}}^{v}$ with the momentum-dependent exciton occupation $N_{\mathrm{A}, Q}^{v}=\left\langle X_{v, Q}^{\dagger} X_{v, Q}\right\rangle$ that we estimate by a thermal Boltzmann distribution in this work. The temperaturedependent valley-specific densities $n_{x}^{v}(T)=\sum_{\boldsymbol{Q}} N_{\mathrm{A}, \boldsymbol{Q}}^{v}$ are illustrated in Fig. 2(b), revealing that $\mathrm{K} \Lambda$ excitons dominate the density at room temperature for monolayer $\mathrm{WSe}_{2}$. This reflects the energetic separation between bright and dark states (cf. Table I in SM) and the three-fold degeneracy of the $\Lambda$ valley [34]. The three-fold degeneracy explicitly enters the exciton distribution, crucially enhancing the occupation of $\mathrm{K} \Lambda$ excitons relative to $\mathrm{KK}$ and $\mathrm{KK}^{\prime}$ excitons.

Applying the second-order Born-Markov approximation [32] (cf. Sec. II B in SM), we find $\dot{n}_{x}=-R_{A} n_{x}^{2}$ with the exciton-exciton annihilation rate coefficient or briefly Auger coefficient $R_{A}$ reading

$$
R_{A}=\frac{2 \pi}{\hbar} \sum_{\substack{\mu, \nu, \rho \\ \boldsymbol{Q}, \boldsymbol{Q}^{\prime}}}\left|W_{\boldsymbol{Q}, \boldsymbol{Q}^{\prime}}^{\mu \nu \rho}\right|^{2} \bar{N}_{\mathrm{A}, \boldsymbol{Q}}^{v}(T) \bar{N}_{\mathrm{A}, \boldsymbol{Q}^{\prime}}^{\rho}(T) \delta(\Delta \epsilon),
$$

with $\bar{N}=N / n_{x}$. The appearing delta function $\delta(\Delta \epsilon)$ ensures that energy is conserved during the scattering process with $\Delta \epsilon=\Delta+\epsilon_{\mathrm{HX}, \boldsymbol{Q}+Q^{\prime}}^{\mu}-\epsilon_{\mathrm{A}, \boldsymbol{Q}}^{\nu}-\epsilon_{\mathrm{A}, \boldsymbol{Q}^{\prime}}^{\rho}$. The detuning $\Delta$ determines the resonance condition for the Auger scattering process that is strongly enhanced for $\Delta \leqslant k_{B} T$. For the intravalley Auger process $\left(\xi_{\mu}=\xi_{v}=\xi_{\rho}=\mathrm{KK}\right)$, the detuning is defined as $\Delta=E_{\mathrm{HX}}-2 E_{\mathrm{A}}$, where $E_{\mathrm{HX}}$ and $E_{\mathrm{A}}$ are exciton resonance energies of the final and initial states, respectively [cf. Fig. 1(b)]. These energies can be directly obtained from recent up-converted photoluminescence measurements for monolayer WSe $\mathrm{With}_{2} E_{\mathrm{HX}}=3.35 \mathrm{eV}$ and $E_{\mathrm{A}}=1.734 \mathrm{eV}$ $[10,17]$. In the case of intervalley EEA processes, the detuning requires the knowledge of binding energies of $\mathrm{HX}$ and
$A$ excitons, which are microscopically calculated by solving the Wannier equation (see Sec. IIC in SM). Furthermore, we approximate the exciton dispersion as parabolic at the considered high-symmetry points, i.e., $\epsilon_{\boldsymbol{Q}}^{\mu}=\frac{\hbar^{2} \boldsymbol{Q}^{2}}{2 M^{\mu}}$ with the total exciton mass $M^{\mu}=m_{e}^{\mu_{e}}+m_{h}^{\mu_{h}}$ and the effective electron (hole) masses $m_{e}^{\mu_{e}}\left(m_{h}^{\mu_{h}}\right)$ being extracted from first-principle calculations [34]. In particular, we note that the effective mass approximation is expected to hold for the final $\mathrm{KK}$ HX state as shown by recent $a b$ initio calculations [10]. Finally, we take into account that excitonic resonances become red-shifted with increasing temperature [35-37]. As a result, we obtain a temperature-dependent detuning, i.e., $\Delta \rightarrow \Delta(T)=\Delta+\Delta_{v}(T)$ with the shift of $\Delta_{v}(T)=\frac{\alpha T^{2}}{T+\beta}$ described by the Varshni model, where the constants $\alpha$ and $\beta$ are extracted from temperature-dependent photoluminescence measurements $[37,38]$.

\section{EXCITON-EXCITON ANNIHILATION RATES}

We evaluate the exciton-exciton annihilation coefficient $R_{A}$ from Eq. (2) for an $\mathrm{hBN}$-encapsulated $\mathrm{WSe}_{2}$ monolayer. We take explicitly into account bright and dark $A$ excitons (KK, $\mathrm{KK}^{\prime}$, and $\left.\mathrm{K} \Lambda\right)$ as initial states and $\mathrm{HX}$ excitons $\left(\mathrm{KK}, \mathrm{KK}^{\prime}\right.$, and $\mathrm{K} \Gamma$ ) up to $n=3 s$ as final states for the Auger scattering process. The overlap of higher-lying states $(n>3 s)$ is negligibly small and thus neglected in the following, cf. Fig. 2(a). In addition to matrix elements and resonance conditions, the efficiency of the Auger process is strongly determined by the distribution of excitons across lower-lying states in thermal equilibrium. Due to the energy splitting between $\mathrm{KK}, \mathrm{KK}^{\prime}$, and $\mathrm{K} \Lambda$ excitons being on the order of tens of meVs, the valley-specific densities strongly change with temperature, as illustrated in Fig. 2(b). The resulting calculated Auger coefficients $R_{A}$ are presented in Fig. 3(a) as a function of temperature.

To test the theoretically predicted Auger-recombination mechanism we take advantage of density-dependent temporally resolved photoluminescence on $\mathrm{hBN}$-encapsulated $\mathrm{WSe}_{2}$ monolayers. At all studied temperatures, the PL transients exhibit a density-dependent increase of the initial decay rate (cf. Fig. S1 in SM) that is well described by the bimolecular recombination law. Being accompanied by the saturation of the total PL intensity, this behavior is characteristic for exciton-exciton annihilation, as was previously demonstrated in the literature [5-8]. At each temperature, we extract the density-induced recombination rate $r_{A}^{\exp }$ as a function of injected exciton density $n_{x}$ at early times after the excitation. The bimolecular coefficient attributed to Auger recombination $R_{A}$ is then determined from the slope, as illustrated in the inset in Fig. 3(b) (cf. Sec. I in SM for details). The potential contributions to the bimolecular recombination rate from biexciton formation $[39,40]$ can be excluded since the biexcitons form much faster $[41,42]$ than the observed density-dependent decay. Furthermore, we emphasize that at the considered densities the average interparticle separation is always smaller than the diffusion length, which should be a mandatory condition for Auger scattering. In the investigated samples, long-lived dark excitons exhibit diffusion lengths on the order of hundreds of nm [43], whereas the exciton-exciton 

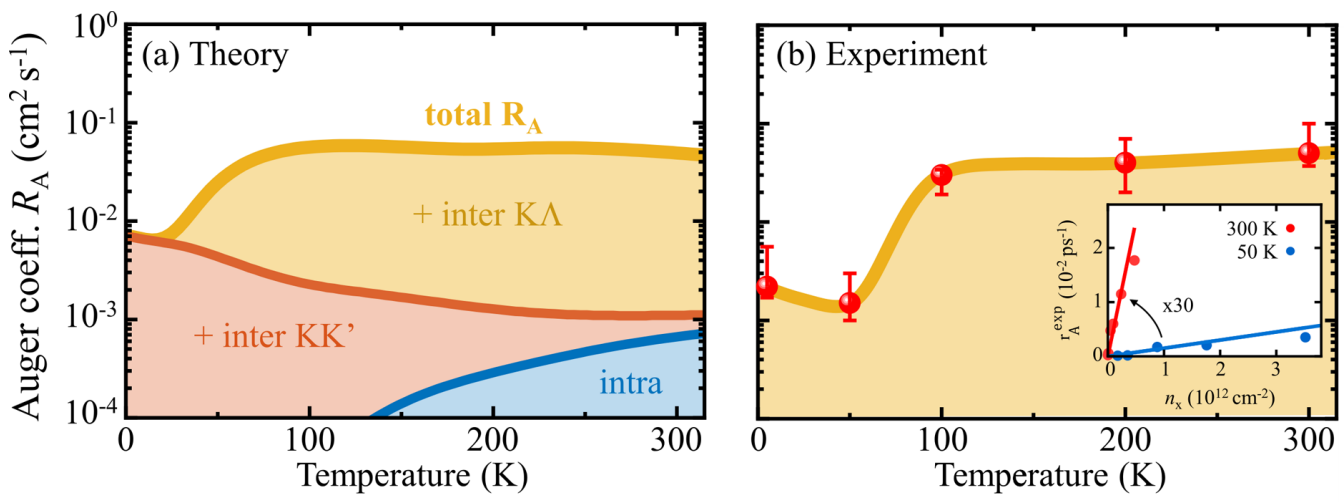

FIG. 3. Temperature dependence of the Auger coefficient $R_{A}$ for hBN-encapsulated WSe 2 . (a) Theoretically calculated $R_{A}$ illustrating the separate contributions of intravalley and intervalley Auger processes (KK, KK', and K $\Lambda$, respectively), cf. Fig. 1(b). We reveal that the recombination of two $\mathrm{K} \Lambda$ excitons is the dominant process for temperatures above $30 \mathrm{~K}$. (b) Experimentally extracted Auger coefficients from time-resolved PL measurements. Error bars represent lower and upper limits for the extracted values. The inset illustrates the density-induced recombination rate $r_{A}^{\text {exp }}$, from which the Auger coefficient can be extracted from the slope.

separation ranges within 30 and $10 \mathrm{~nm}$ for the studied densities between $10^{11}$ and $10^{12} \mathrm{~cm}^{-2}$.

Experimentally obtained, temperature-dependent Auger coefficients are presented in Fig. 3(b) in direct comparison to the theoretical predictions in Fig. 3(a). Both, in theory and experiment the Auger coefficient increases by an order of magnitude when increasing the temperature in the range of 50 to $100 \mathrm{~K}$ and remains nearly constant up to room temperature. From microscopic calculations we obtain $R_{A}=0.005 \mathrm{~cm}^{2} / \mathrm{s}$ and $0.05 \mathrm{~cm}^{2} / \mathrm{s}$ at $T=10 \mathrm{~K}$ and $T=300 \mathrm{~K}$, respectively. These values match (without fitting) the experimentally determined coefficients of $0.001-0.006 \mathrm{~cm}^{2} / \mathrm{s}$ at $\mathrm{T} \leqslant 50 \mathrm{~K}$ and $0.04-0.1 \mathrm{~cm}^{2} / \mathrm{s}$ at $300 \mathrm{~K}$. The obtained quantitative agreement between theory and experiment strongly supports both the predominant role of the dark excitons and the $n=1 \mathrm{HX}$ final states for Auger recombination.

To understand the microscopic origin of the drastic increase of the Auger coefficients as a function of temperature, we explicitly separate the contributions from intra and intervalley Auger scattering in theory [corresponding to the processes I, II, and III illustrated in Fig. 1(b)]. We find that the intervalley $\mathrm{KK}^{\prime}$ and $\mathrm{K} \Lambda$ Auger scattering involving the momentum-dark $\mathrm{KK}^{\prime}$ and $\mathrm{K} \Lambda$ excitons clearly dominate the Auger coefficient $R_{A}$ for low and high temperatures, respectively [red and orange region in Fig. 3(a)]. The intravalley Auger scattering involving the bright KK excitons is negligible (blue region, note the logarithmic scale). This is a consequence of the spectral ordering of exciton states in $\mathrm{WSe}_{2}$ monolayers, where the momentum-dark $\mathrm{KK}^{\prime}$ excitons are the energetically lowest states followed by the dark $\mathrm{K} \Lambda$ excitons and finally the bright KK states. The relative position of these exciton states has been determined microscopically by solving the exciton Wannier equation (see SM for more details). As a result, the dark states carry by far the largest occupation and thus dominate the Auger scattering processes, despite smaller values of the Auger matrix elements.

The obtained temperature dependence of the Auger coefficient thus strongly reflects the changes in the valley-specific exciton densities $n_{x}^{v}(T)$ with $v=\mathrm{KK}, \mathrm{KK}^{\prime}, \mathrm{K} \boldsymbol{\Lambda}$, cf. Fig. 2(b). At low temperatures up to $20 \mathrm{~K}$ the $\mathrm{KK}^{\prime}$ intervalley Auger process [red area in Fig. 3(a)] is the predominant scattering channel. The K $\Lambda$ Auger scattering takes over from $20 \mathrm{~K}$ and quickly becomes, by far, the most prominent contribution [orange area in Fig. 3(a)]. The sharp increase in the Auger coefficient between $50-100 \mathrm{~K}$ reflects the predominant population of the three-fold degenerate $\mathrm{K} \Lambda$ state at intermediate temperatures [cf. Fig. 2(a)]. Furthermore, the Auger matrix element is much more efficient for $\mathrm{K} \Lambda$ than $\mathrm{KK}^{\prime}$ intervalley scattering due to the larger momentum transfer involved in the later process suppressing the interaction [cf. Fig. 2(b)]. For $T>100 \mathrm{~K}$, only minor changes in the relative exciton distributions result in approximately constant Auger coefficients at higher temperatures.

Similar to the influence of temperature, the overall efficiency of the excitonic Auger scattering should also strongly depend on the dielectric environment that modifies the relative energies of the involved excitonic states. Recently, a strong suppression of the Auger recombination in $\mathrm{hBN}$-encapsulated TMD monolayers was experimentally demonstrated $[22,44]$. The theoretical approach outlined above now allows us to analyze and reveal the microscopic origin of this effect. In the following, we investigate the impact of the dielectric environment on the exciton-exciton annihilation rate and study, in particular, the case of hBN-encapsulated samples versus samples placed on the standard $\mathrm{SiO}_{2}$ substrate.

In Fig. 4(a), we illustrate the combined temperature and substrate dependence of the Auger coefficient $R_{A}$. There are two distinct trends: (i) For a fixed temperature, Auger scattering becomes less efficient with the dielectric screening and (ii) the Auger coefficients show a maximum at a certain substrate-dependent temperature. The first trend is further shown in Fig. 4(b), where we consider the screening dependence of Auger coefficients for three different temperatures. The reduction of Auger scattering with screening is observed at all temperatures. Comparing the two most common dielectric environments, $\mathrm{SiO}_{2}\left(\epsilon_{s} \approx \frac{3.9+1}{2}=2.45\right)$ and hBN-encapsulation $\left(\epsilon_{s}=4.5\right)$, we find $R_{A, \mathrm{SiO}_{2}}=$ $0.13 \mathrm{~cm}^{2} / \mathrm{s}$ vs $R_{A, \mathrm{hBN}}=0.05 \mathrm{~cm}^{2} / \mathrm{s}$ at room temperature, i.e., we predict a reduction of the Auger coefficient by approximately $60 \%$ in the case of $\mathrm{hBN}$-encapsulated $\mathrm{WSe}_{2}$. This reduction can partly be attributed to a weakened Coulomb interaction with screening, but importantly it 


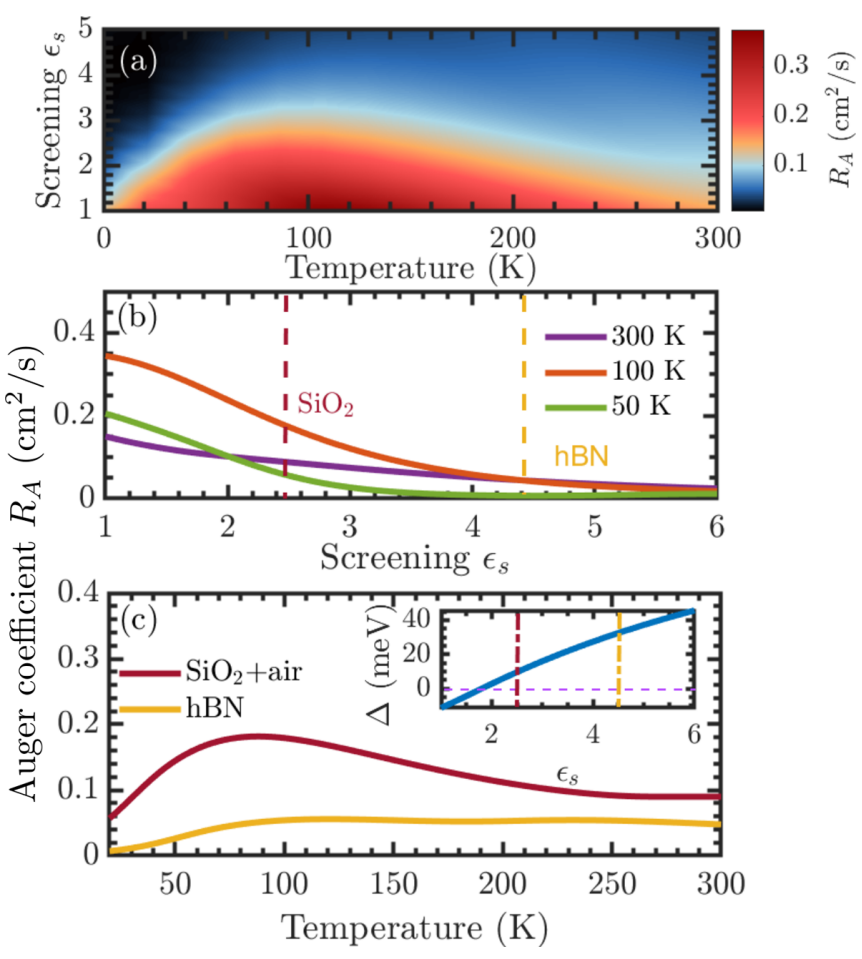

FIG. 4. Substrate and temperature dependence of the Auger coefficient $R_{A}$ in monolayer $\mathrm{WSe}_{2}$ from microscopic theory. (a) $R_{A}$ as a function of screening and temperature, revealing a nonmonotonic temperature behavior. (b) Screening dependence of $R_{A}$ for different temperatures $T=50,100$, and $300 \mathrm{~K}$. (c) Temperature-dependent Auger coefficients in the case of hBN-encapsulation $\left(\epsilon_{s}=4.5\right)$ and for the $\mathrm{SiO}_{2}$ substrate $\left(\epsilon_{s}=2.45\right)$. As illustrated in the inset for the $\mathrm{K} \Lambda$ Auger process, the increase in screening from $\epsilon_{s}=2.45$ to $\epsilon_{s}=4.5$ makes the Auger scattering more off-resonant leading to a less efficient $R_{A}$ in hBN-encapsulated TMDs.

is also a consequence of quenched resonance conditions determined by the detuning $\Delta$, cf. Eq. (2). To further quantify these effects, we determine the decrease in the Auger matrix element between samples on a $\mathrm{SiO}_{2}$ substrate and $\mathrm{hBN}$-encapsulated samples for the predominant $\mathrm{K} \Lambda$ Auger scattering channel to be approximately $30 \%$ in hBN-encapsulated $\mathrm{WSe}_{2}$ monolayers.

To understand how the resonance conditions change with the substrate we investigate the screening dependence of the individual components entering the detuning. The $A$ resonance energy (initial state) is known to only weakly vary with dielectric screening due to the simultaneous reduction of the band-gap and the exciton binding energy $[45,46]$ and therefore it can be assumed to be approximately constant. Moreover, the energy splittings between different conduction bands are expected to be to a large extent independent of screening since the Coulomb renormalization only affects the absolute energies of the bands [47]. The detuning for the dominant $\mathrm{K} \Lambda$-Auger channel at room temperature acquires a weak screening dependence [cf. the inset of Fig. 4(c)], stemming from the different screening-induced changes in intra and intervalley binding energies. We predict $\Delta_{\mathrm{hBN}} \approx$ $2 \Delta_{\mathrm{SiO}_{2}}=30 \mathrm{meV}$ for the $\mathrm{K} \Lambda$ Auger scattering channel with the dark $1 s \mathrm{~K} \Gamma \mathrm{HX}$ exciton as final state [cf. Fig. 1(b)] resulting in weaker Auger coefficients for $\mathrm{hBN}$-encapsulated samples. The contribution from higher-order exciton states $(n>1 s)$ to the Auger rates is seen to be suppressed due to large detunings (e.g., $|\Delta|_{2 s, \mathrm{hBN}} \approx 200 \mathrm{meV}$ ). This in combination with the weakened Auger matrix elements is the origin of the previous experimental observations showing strongly quenched Auger scattering for hBN-encapsulated TMDs $[22,44]$. Note that defect-assisted Auger scattering might also play a role for TMDs on a $\mathrm{SiO}_{2}$ substrate due to disorder, while it is expected to be negligible in the case of hBN-encapsulation.

Finally, we demonstrate an intriguing nonmonotonic temperature dependence of Auger scattering for fixed dielectric screening in the case of $\mathrm{SiO}_{2}$ and $\mathrm{hBN}$-encapsulation, cf. Fig. 4(c). Interestingly, we find a clear maximum in the Auger coefficient for $\mathrm{WSe}_{2}$ on $\mathrm{SiO}_{2}$ at around $80 \mathrm{~K}$. The Auger coefficient $R_{A}$ can be approximated by $R_{A} \approx \frac{1}{k_{B} T} \exp \left[-|\Delta| /\left(k_{B} T\right)\right]$, displaying a maximum at $T_{\max }=|\Delta| / k_{B}$ [18]. This approximate expression becomes exact in the limit of a constant Auger matrix element and when a single exciton species $v=\rho \equiv v_{0}$ dominates the Auger coefficient. The approximation allows us to understand the temperature dependence of the Auger coefficients, which is determined by the initial $A$ exciton distribution and the availability of initial and final states fulfilling the conservation of energy and momentum, cf. Eq. (2). For very low temperatures the exciton distribution is strongly localized at vanishing kinetic energies and hence, the EEA is inefficient due to the nonzero energy detuning of initial and final states. With increasing temperature the momentum-dependent distribution becomes broadened, facilitating energy and momentum conservation, and thus offering additional scattering channels leading to an enhanced $R_{A}$. A further increase in temperature leads to a redistribution of excitons and an overall decrease in the initial population of exciton states, resulting in a reduction of the Auger coefficients. The interplay of those two effects is the origin of the observed maximum in the $R_{A}$ at certain intermediate temperatures. In the case of hBN-encapsulated samples we do not observe a pronounced maximum of the Auger coefficient as a function of temperature, as the resonance energy cannot be reached in the considered temperature range, i.e., $|\Delta|>k_{B} T$ at all temperatures. Here, the large scattering efficiency at higher temperatures is solely determined by the $\mathrm{K} \Lambda$ exciton occupation.

\section{CONCLUSION}

In this joint theory-experiment study combining microscopic, material-specific modeling with time-resolved photoluminescence measurements, we establish a fundamental understanding of Auger-like exciton-exciton annihilation processes in atomically thin semiconductors. We demonstrate the key importance of dark intervalley excitons in the prototypical $\mathrm{WSe}_{2}$ material resulting in an intriguing temperature dependence of Auger processes. We find an excellent qualitative and quantitative agreement between theory and experiment without any adjusted free parameters. Our results also contribute to resolving an open question in the literature regarding the origin of consistently observed suppression 
of exciton-exciton annihilation upon hBN-encapsulation. Overall, our work provides microscopic insights into the many-particle processes behind the technologically important exciton-exciton annihilation channels in atomically thin semiconductors. The developed approach can be further generalized to van der Waals heterostructures and twisted moiré exciton systems.

\section{ACKNOWLEDGMENTS}

We thank Maja Feierabend (Chalmers) and Paulo Eduardo de Faria Junior and Kai-Qiang Lin (University of Regensburg) for fruitful discussions. This project received funding from
Deutsche Forschungsgemeinschaft via CRC 1083 (Project No. B09), Emmy Noether Initiative (CH 1672/1, ProjectID 287022282), CRC 1277 (Project-ID 314695032, Project No. B05), CRC 953 (Project No. B13), the European Unions Horizon 2020 research and innovation program under Grant agreement No. 881603 (Graphene Flagship) and the Würzburg-Dresden Cluster of Excellence on Complexity and Topology in Quantum Matter ct.qmat (EXC 2147, Project-ID: 390858490). Furthermore, we are thankful to Vinnova for the support via the 2D-TECH competence center. K.Watanabe and T.T. acknowledge support from the Elemental Strategy Initiative conducted by the MEXT, Japan (Grant No. JPMXP0112101001) and JSPS KAKENHI (Grants No. JP19H05790 and No. JP20H00354).
[1] G. Wang, A. Chernikov, M. M. Glazov, T. F. Heinz, X. Marie, T. Amand, and B. Urbaszek, Rev. Mod. Phys. 90, 021001 (2018).

[2] T. Mueller and E. Malic, npj 2D Mater. Appl. 2, 29 (2018).

[3] Y. Cao, V. Fatemi, S. Fang, K. Watanabe, T. Taniguchi, E. Kaxiras, and P. Jarillo-Herrero, Nature (London) 556, 43 (2018).

[4] P. Merkl, F. Mooshammer, P. Steinleitner, A. Girnghuber, K.-Q. Lin, P. Nagler, J. Holler, C. Schüller, J. M. Lupton, T. Korn et al., Nat. Mater. 18, 691 (2019).

[5] D. Sun, Y. Rao, G. A. Reider, G. Chen, Y. You, L. Brezin, A. R. Harutyunyan, and T. F. Heinz, Nano Lett. 14, 5625 (2014).

[6] N. Kumar, Q. Cui, F. Ceballos, D. He, Y. Wang, and H. Zhao, Phys. Rev. B 89, 125427 (2014).

[7] L. Yuan and L. Huang, Nanoscale 7, 7402 (2015).

[8] Y. Yu, Y. Yu, C. Xu, A. Barrette, K. Gundogdu, and L. Cao, Phys. Rev. B 93, 201111(R) (2016).

[9] M. Manca, M. Glazov, C. Robert, F. Cadiz, T. Taniguchi, K. Watanabe, E. Courtade, T. Amand, P. Renucci, X. Marie et al., Nat. Commun. 8, 14927 (2017).

[10] K.-Q. Lin, C. S. Ong, S. Bange, P. E. Faria Junior, B. Peng, J. D. Ziegler, J. Zipfel, C. Bäuml, N. Paradiso, K. Watanabe et al., Nat. Commun. 12, 5500 (2021).

[11] K.-Q. Lin, S. Bange, and J. M. Lupton, Nat. Phys. 15, 242 (2019).

[12] J.-H. Kim, M. R. Bergren, J. C. Park, S. Adhikari, M. Lorke, T. Frauenheim, D.-H. Choe, B. Kim, H. Choi, T. Gregorkiewicz et al., Nat. Commun. 10, 5488 (2019).

[13] T. Winzer and E. Malić, Phys. Rev. B 85, 241404(R) (2012).

[14] T. Winzer, A. Knorr, and E. Malic, Nano Lett. 10, 4839 (2010).

[15] D. Brida, A. Tomadin, C. Manzoni, Y. J. Kim, A. Lombardo, S. Milana, R. R. Nair, K. S. Novoselov, A. C. Ferrari, G. Cerullo et al., Nat. Commun. 4, 1987 (2013).

[16] F. Wendler and E. Malic, in Terahertz Emitters, Receivers, and Applications VI (International Society for Optics and Photonics, San Diego, 2015), Vol. 9585, p. 95850D.

[17] K.-Q. Lin, P. E. F. Junior, J. M. Bauer, B. Peng, B. Monserrat, M. Gmitra, J. Fabian, S. Bange, and J. M. Lupton, Nat. Commun. 12, 1553 (2021).

[18] B. Han, C. Robert, E. Courtade, M. Manca, S. Shree, T. Amand, P. Renucci, T. Taniguchi, K. Watanabe, X. Marie, L. E. Golub, M. M. Glazov, and B. Urbaszek, Phys. Rev. X 8, 031073 (2018).
[19] A. Steinhoff, F. Jahnke, and M. Florian, Phys. Rev. B 104, 155416 (2021).

[20] M. Massicotte, F. Vialla, P. Schmidt, M. B. Lundeberg, S. Latini, S. Haastrup, M. Danovich, D. Davydovskaya, K. Watanabe, T. Taniguchi et al., Nat. Commun. 9, 1633 (2018).

[21] M. Danovich, V. Zólyomi, V. I. Fal'ko, and I. L. Aleiner, 2D Mater. 3, 035011 (2016).

[22] Y. Hoshi, T. Kuroda, M. Okada, R. Moriya, S. Masubuchi, K. Watanabe, T. Taniguchi, R. Kitaura, and T. Machida, Phys. Rev. B 95, 241403(R) (2017).

[23] J. Zipfel, M. Kulig, R. Perea-Causín, S. Brem, J. D. Ziegler, R. Rosati, T. Taniguchi, K. Watanabe, M. M. Glazov, E. Malic, and A. Chernikov, Phys. Rev. B 101, 115430 (2020).

[24] T. Deilmann and K. S. Thygesen, Phys. Rev. B 96, 201113(R) (2017).

[25] E. Malic, M. Selig, M. Feierabend, S. Brem, D. Christiansen, F. Wendler, A. Knorr, and G. Berghäuser, Phys. Rev. Mater. 2, 014002 (2018).

[26] J. Madéo, M. K. Man, C. Sahoo, M. Campbell, V. Pareek, E. L. Wong, A. Al-Mahboob, N. S. Chan, A. Karmakar, B. M. K. Mariserla et al., Science 370, 1199 (2020).

[27] R. Wallauer, R. Perea-Causin, L. Münster, S. Zajusch, S. Brem, J. Güdde, K. Tanimura, K.-Q. Lin, R. Huber, E. Malic et al., Nano Lett. 21, 5867 (2021).

[28] S. Brem, A. Ekman, D. Christiansen, F. Katsch, M. Selig, C. Robert, X. Marie, B. Urbaszek, A. Knorr, and E. Malic, Nano Lett. 20, 2849 (2020).

[29] D. Erkensten, S. Brem, and E. Malic, Phys. Rev. B 103, 045426 (2021).

[30] See Supplemental Material at http://link.aps.org/supplemental/ 10.1103/PhysRevB.104.L241406 for additional details on the temperature- and time-resolved photoluminescence measurements, microscopic modeling (derivation of excitonic Auger matrix elements, exciton-exciton annihilation rates, and exciton binding energies) and $a b$ initio calculations; also see references [48-72] therein.

[31] H. Haug and S. W. Koch, Quantum Theory of the Optical and Electronic Properties of Semiconductors (World Scientific, Singapore, 2009).

[32] M. Kira and S. W. Koch, Prog. Quantum. Electron. 30, 155 (2006).

[33] G. Berghäuser and E. Malic, Phys. Rev. B 89, 125309 (2014). 
[34] A. Kormányos, G. Burkard, M. Gmitra, J. Fabian, V. Zólyomi, N. D. Drummond, and V. Fal'ko, 2D Mater. 2, 022001 (2015).

[35] Y. P. Varshni, Physica 34, 149 (1967).

[36] K. O’Donnell and X. Chen, Appl. Phys. Lett. 58, 2924 (1991).

[37] A. Arora, M. Koperski, K. Nogajewski, J. Marcus, C. Faugeras, and M. Potemski, Nanoscale 7, 10421 (2015).

[38] A. Arora, K. Nogajewski, M. Molas, M. Koperski, and M. Potemski, Nanoscale 7, 20769 (2015).

[39] Y. You, X. Zhang, T. C. Berkelbach, M. S. Hybertsen, D. R. Reichman, and T. F. Heinz, Nat. Phys. 11, 477 (2015).

[40] Z. Ye, L. Waldecker, E. Y. Ma, D. Rhodes, A. Antony, B. Kim, X.-X. Zhang, M. Deng, Y. Jiang, Z. Lu, D. Smirnov, K. Watanabe, T. Taniguchi, J. Hone, and T. F. Heinz, Nat. Commun. 9, 3718 (2018).

[41] A. Steinhoff, M. Flrian, A. Singh, K. Tran, M. Kolarczik, S. Helmrich, A. W. Achtstein, U. Woggon, N. Owschimikow, F. Jahnke, and X. Li, Nat. Phys. 14, 1199 (2018).

[42] P. Nagler, M. V. Ballottin, A. A. Mitioglu, M. V. Durnev, T. Taniguchi, K. Watanabe, A. Chernikov, C. Schüller, M. M. Glazov, P. C. M. Christianen, and T. Korn, Phys. Rev. Lett. 121, 057402 (2018).

[43] K. Wagner, J. Zipfel, R. Rosati, E. Wietek, J. D. Ziegler, S. Brem, R. Perea-Causín, T. Taniguchi, K. Watanabe, M. M. Glazov, E. Malic, and A. Chernikov, Phys. Rev. Lett. 127, 076801 (2021).

[44] M. Kulig, J. Zipfel, P. Nagler, S. Blanter, C. Schüller, T. Korn, N. Paradiso, M. M. Glazov, and A. Chernikov, Phys. Rev. Lett. 120, 207401 (2018).

[45] S. Lippert, L. M. Schneider, D. Renaud, K. N. Kang, O. Ajayi, J. Kuhnert, M.-U. Halbich, O. M. Abdulmunem, X. Lin, K. Hassoon et al., 2D Mater. 4, 025045 (2017).

[46] W.-T. Hsu, J. Quan, C.-Y. Wang, L.-S. Lu, M. Campbell, W.-H. Chang, L.-J. Li, X. Li, and C.-K. Shih, 2D Mater. 6, 025028 (2019).

[47] L. Waldecker, A. Raja, M. Rösner, C. Steinke, A. Bostwick, R. J. Koch, C. Jozwiak, T. Taniguchi, K. Watanabe, E. Rotenberg, T. O. Wehling, and T. F. Heinz, Phys. Rev. Lett. 123, 206403 (2019).

[48] P. Giannozzi, S. Baroni, N. Bonini, M. Calandra, R. Car, C. Cavazzoni, D. Ceresoli, G. L. Chiarotti, M. Cococcioni, I. Dabo, A. D. Corso, S. de Gironcoli, S. Fabris et al., J. Condens. Matter Phys. 21, 395502 (2009).

[49] A. Marini, C. Hogan, M. Grüning, and D. Varsano, Comput. Phys. Commun. 180, 1392 (2009).
[50] J. Deslippe, G. Samsonidze, D. A. Strubbe, M. Jain, M. L. Cohen, and S. G. Louie, Comput. Phys. Commun. 183, 1269 (2012).

[51] S. Grimme, S. Ehrlich, and L. Goerigk, J. Comput. Chem 32, 1456 (2011).

[52] F. Bruneval and X. Gonze, Phys. Rev. B 78, 085125 (2008).

[53] D. R. Hamann, Phys. Rev. B 88, 085117 (2013).

[54] R. Gillen and J. Maultzsch, Phys. Rev. B 97, 165306 (2018).

[55] J. P. Perdew, K. Burke, and M. Ernzerhof, Phys. Rev. Lett. 77, 3865 (1996).

[56] E. Liu, J. van Baren, T. Taniguchi, K. Watanabe, Y.-C. Chang, and C. H. Lui, Phys. Rev. Research 1, 032007(R) (2019).

[57] L. V. Keldysh, J. Exp. Theor. Phys. 29, 658 (1979).

[58] E. O. Kane, J. Phys. Chem. Solids 1, 249 (1957).

[59] J. M. Luttinger and W. Kohn, Phys. Rev. 97, 869 (1955).

[60] S. Brem, J. Zipfel, M. Selig, A. Raja, L. Waldecker, J. D. Ziegler, T. Taniguchi, K. Watanabe, A. Chernikov, and E. Malic, Nanoscale 11, 12381 (2019).

[61] M. He, P. Rivera, D. Van Tuan, N. P. Wilson, M. Yang, T. Taniguchi, K. Watanabe, J. Yan, D. G. Mandrus, H. Yu, H. Dery, W. Yao, and X. Xu, Nat. Commun 11, 618 (2020).

[62] A. L. Ivanov and H. Haug, Phys. Rev. B 48, 1490 (1993).

[63] P. Cudazzo, I. V. Tokatly, and A. Rubio, Phys. Rev. B 84, 085406 (2011).

[64] S. Latini, T. Olsen, and K. S. Thygesen, Phys. Rev. B 92, 245123 (2015).

[65] M. L. Trolle, T. G. Pedersen, and V. Véniard, Sci. Rep. 7, 39844 (2017).

[66] D. Y. Qiu, F. H. da Jornada, and S. G. Louie, Phys. Rev. B 93, 235435 (2016).

[67] F. H. da Jornada, D. Y. Qiu, and S. G. Louie, Phys. Rev. B 95, 035109 (2017).

[68] D. V. Rybkovskiy, I. C. Gerber, and M. V. Durnev, Phys. Rev. B 95, 155406 (2017).

[69] F. Katsch, M. Selig, A. Carmele, and A. Knorr, Phys. Status Solidi B 255, 1800185 (2018).

[70] F. Katsch, M. Selig, and A. Knorr, Phys. Rev. Lett. 124, 257402 (2020).

[71] A. A. Mosto, J. R. Yates, G. Pizzi, Y.-S. Lee, I. Souza, D. Vanderbilt, and N. Marzari, Comput. Phys. Commun. 185, 2309 (2014).

[72] A. Raja, L. Waldecker, J. Zipfel, Y. Cho, S. Brem, J. D. Ziegler, M. Kulig, T. Taniguchi, K. Watanabe, E. Malic, T. F. Heinz, T. C. Berkelbach, and A. Chernikov, Nat. Nanotechnol. 14, 832 (2019). 